\title{
Use of Salivary Biomarkers for Diagnosis of Periodontal Disease Activity: A Literature Review
}

\author{
Fernanda Regina Godoy Rocha, Rafael Scaf de Molon, Mateus Rodrigues Tonetto, Shelon Cristina Souza Pinto \\ Eduardo Mariscal Munoz, Jose Eduardo Cesar Sampaio
}

\begin{abstract}
Periodontal disease is an infectious disease characterized by the connective tissue destruction and consequent alveolar bone loss in response to plaque accumulation on the tooth surface. The clinical diagnosis of periodontal disease is based both on clinical examination involving the evaluation of probing depth and radiographic examination of alveolar bone loss but these examinations are not enough to determine the activity of the disease process. For that reason, it has been proposed to seek predictive disease markers in an attempt to assess the disease activity and so, evaluate the efficacy of the periodontal disease treatment. The aim of this review is to present recent advances in the development of proteomic, genomics and microbial biomarkers and potential clinical applications. It was concluded that periodontal treatment based on assessing the levels of salivary biomarkers emerges as a promising method in near future and will become an integral part of the evaluation of periodontal health.
\end{abstract}

Keywords: Salivary glands, Periodontal disease, Oral diagnosis, Periodontitis.

How to cite this article: Rocha FRG, de Molon RS, Tonetto MR, Souza Pinto SC, Munoz EM, Sampaio JEC. Use of Salivary Biomarkers for Diagnosis of Periodontal Disease Activity: A Literature Review. World J Dent 2013;4(4):250-255.

Source of support: Nil

Conflict of interest: None declared

\section{INTRODUCTION}

Periodontal disease is a chronic bacterial infection characterized by the destruction of connective tissue and consequent alveolar bone loss in response to biofilm accumulation on the tooth surface. ${ }^{1,2}$ The process can be restricted to the gingival tissues and are called gingivitis, or may affect the supporting tissues of the teeth, being called periodontitis. In cases of gingivitis that progress to periodontitis, local tissue destruction by the defense cells of the host immune response causes periodontal tissues loss such as periodontal ligament, connective tissue and alveolar bone. ${ }^{3}$

The clinical diagnosis of periodontal disease is traditionally based on clinically examination of the detectable parameters such as probing depth, attachment level, plaque index, bleeding on probing and radiographic assessment of alveolar bone loss. These are informative to assess the severity of the disease, but not yet fully determinants for evaluation of their activity. The presence of bleeding on probing is still the best available parameter for evaluation of disease activity. On the other hand, bleeding on probing is not specific enough and may reveal false positive results. ${ }^{4}$ Thus, it has been proposed to seek predictive markers of disease (predictive testing) as the assessment of disease activity and treatment efficacy (diagnostic tests) for periodontal disease. ${ }^{3,5}$

The identification of sites or individuals susceptible to periodontal disease and the diagnosis of its active phase is still a challenge for clinicians and researchers, often with results that are difficult to be compared from one study to another. Thus, the possibility has been demonstrated that a simple and accurate diagnostic tool, the use of salivary biomarkers could provide more accurate and allow more reliable evaluation periodontal disease state. ${ }^{6}$

\section{LITERATURE REVIEW}

\section{Saliva as a Diagnostic Tool}

Saliva is a biological fluid critical for maintaining the health of the oral cavity. Their physicochemical and biochemical properties, with its complex composition, make this fluid a compound with multiple functions, including the antibacterial, antiviral and antifungal properties, buffering capacity, activity during chewing through the activation of enzymes (amylase, protease and nuclease), presence of mineralizing agents for protection and repair of hard tissues, lubricating properties, reparative and protective to the gingival tissues.

Saliva is a hypotonic fluid, comprising $95.5 \%$ water and $0.5 \%$ of ions such as calcium, potassium, sodium, and organic molecules such as histatins, cystatins, defensins, lysozymes, aminoperoxidase, lactoferrin, secretory immunoglobulins, mucin, lipids and others.

The collection of saliva samples for analysis can be done easily with the use of stimulating agents or with no stimulation. The unstimulated saliva has been most frequently used in diagnostics, as the stimulated has a reduced concentration of biomarkers, which may hinder their detection. ${ }^{5}$ Salivary secretion into the buccal cavity occurs from the salivary glands into the oral cavity at a flow between 1.5 and $2.0 \mathrm{ml} / \mathrm{min}, 50 \%$ of which are from the parotid gland, $35 \%$ of the submandibular gland, 7 to $8 \%$ of sublingual gland and other 7 to $8 \%$ smaller glands. For possible diagnosis of systemic diseases, the total saliva 
are used, which is a mixture of secretions from the salivary glands with an increase of substances derived from the gingival crevicular fluid, epithelial cells, bronchial or nasal secretions, microorganisms and its metabolic products, or food scraps. ${ }^{7}$

The main reason given for the use of saliva as a diagnostic tool is that the majority of biomarkers present in blood and urine may also be found in saliva samples. Salivary biomarkers have already been used for the diagnosis of systemic diseases, such as C-reactive protein (CRP) and immunoglobulin- $\mathrm{A}^{8}$ (IgA), cardiovascular diseases, sodium nitrate, uric acid, amylase, lactoferrin for kidney diseases, cancer diseases, ${ }^{9}$ for the detection of oral squamous cell carcinoma, ${ }^{10}$ and nitric oxide. ${ }^{11}$ Some products of oxidative reactions associated with the presence of diabetes, which are found in the blood, have also been detected in the saliva, as well as antibodies that occur for markers may be of some autoimmune diseases. Infectious diseases can also have their causative agents such as some viruses, fungi and bacteria identified in saliva. ${ }^{5,6,12-15}$

Due to the ease and quickly data collection, through noninvasive method and easy storage of samples, there has been great interest in investigating the biomarkers present in saliva, soluble specific biological markers that could be associated with the presence and/or activity of periodontal disease. Among them, are some enzymes (alkaline phosphatase, collagenase, aminopeptidase, esterase, elastase) immunoglobulins ( $\operatorname{Ig} \mathrm{A}, \operatorname{IgG}, \operatorname{IgM}$ ), some cytokines and other proteins (fibronectin, cystatins, lactoferrin, growth factors vascular endothelial and platelet-activating) calcium ions and hormones, such as cortisol. ${ }^{6,7}$ Thus, this review aims to summarize the recent advances in the identification of proteomic, genomic and microbial biomarkers and their possible clinical applications.

\section{Salivary Biomarkers Specific to Periodontal Disease}

In periodontal disease, bacterial products trigger the immune response and, from this, there are the activation of polymorphonuclear neutrophils, monocytes and macrophages, with involvement of these cells to the site and release of cytokines, such as prostaglandin (PGE2), tumor necrosis factor (TNF), and interleukins (IL-1 and IL-6), leading to the extent of the inflammatory process. Consequently, they produced matrix metalloproteinases (MMPs) that are collagen destructive enzymes. MMP-8 is considered the most destructive of MMPs, causing extracellular matrix degradation and has been identified as an important enzyme in periodontal disease destruction. ${ }^{16}$

\section{Microbial and Genomic Biomarkers}

Previous study ${ }^{17}$ had been detected a high level of MMP-8 in saliva of individuals with periodontitis compared to healthy patients. ${ }^{17}$ Salivary levels of MMP-1 were similar in both groups and the high concentration of MMP-8 was found and assigned to a state of periodontal disease. This parameter has been used as a possible indicator of the periodontitis activity. ${ }^{18-20}$ Another think to consider is that the identification of MMPs in saliva, as an indicative that MMPs are expressed in periodontal disease, considering that they would be present only where there was collagen destruction, a condition found in active periodontal disease. However, this is not the only event involved in the disease activity and, even if the MMPs are present, there is no way to ensure its detection in saliva samples. Thus, the high concentration of MMPs cannot be considered solely as a diagnosis of active periodontal disease.

In order to seek an association between the presence of specific enzymes and cytokines with the activity of periodontal pathogens, a study ${ }^{21}$ evaluated the levels of enzymes present in saliva, such as aspartate aminotransferase (AST), alanine aminotransferase and lactate dehydrogenase (LDH) after scaling and root planning (SRP), and also to clarify the genotype of IL-1 in enzyme levels. The saliva stimulated by the patient chewed paraffin for 5 minutes was collected at the initial period and 4 weeks after SRP. Then, the authors assessed the levels of AST, ALT and LDH and the DNA extraction was taken, from which was determined the genotype of IL-1. All enzymes evaluated reduced significantly after SRP. The allele of IL-1 evaluated, however, did not influence the reduction of clinical parameters, although it is suggested that may have influenced the values of salivary AST and ALT.

A clinical study ${ }^{22}$ attended subjects divided into a group of periodontally healthy or with gingivitis and another group with mild or severe periodontal disease, also sought to measure the levels of proinflammatory cytokines and markers of bone resorption and bone turnover, with the identification of some periodontal pathogens, aiming to relate them to other markers. The authors used a diagnostic test to characterize the early stages of periodontal infection and disease progression. Data from salivary markers were correlated with clinical, radiographic, and also the levels of plaque biofilm and evaluated using PCR, in an attempt to generate models to identify periodontal disease. Confirming the results already described for gingival crevicular fluid, biomarkers such as MMP-8 and MMP-9 were found elevated in subjects with periodontitis. ${ }^{22}$ Combining the presence of multiple associations of salivary biomarkers (such as MMP8 and 9 and OPG, for example) to anaerobic periodontal pathogens of the red complex ( $P$. gingivalis and T. denticola), the authors suggest the usefulness of salivary biomarkers monitoring during the host response to an oral disease, especially by means of rapid diagnostic tests. ${ }^{23}$ 


\section{Evaluation of Proteomic Biomarkers}

These analyzes seek to compare the proteomic profile of patients with periodontitis with the profile of periodontally healthy subjects, evaluating distinctions between profiles, from changes of salivary proteins in the presence of periodontal inflammation, suggesting that this could contribute to the improvement in the diagnostic of periodontal diseases. ${ }^{24,25}$

Significant increases in the salivary levels of inflammatory cytokines such as TNF- $\alpha$, have been found significantly elevated in patients with periodontal disease $(4.33 \mathrm{pg} / \mathrm{ml})$ than in periodontally healthy individuals $(2.03 \mathrm{pg} / \mathrm{ml})$, with maximum level reaching $27.96 \mathrm{pg} / \mathrm{ml}$ in subjects with periodontitis. These dates were correlated with clinical findings, and individuals with TNF- $\alpha$ salivary levels above $5.75 \mathrm{pg} / \mathrm{ml}$ had significantly more sites with bleeding on probing. ${ }^{25}$

The correlation between others specifics biomarkers to periodontal disease and their clinical characteristics has been evaluated by means of three key aspects of its pathogenic processes: inflammation, collagen degradation and bone resorption. In a case-control study, there was a link between periodontal disease and levels of salivary IL-1 $\beta$, MMP- 8 and osteoprotegerin (OPG). Levels of IL-1 $\beta$ and MMP- 8 found in the saliva of periodontitis patients were significantly higher than in the control group, and suggested that these could actually be used as biomarkers of periodontitis, and the qualitative changes in the composition of salivary biomarkers could be significant in relation to the diagnosis and treatment of periodontal diseases. ${ }^{19}$

Faced with the proposition that levels of salivary biomarkers could be used to distinguish periodontally healthy individuals from periodontitis patient, Teles et $\mathrm{al}^{26}$ sought to determine possible differences between the levels of 10 different cytokines present in the saliva samples of a group of periodontally healthy subjects and another group with chronic periodontitis, correlating the concentrations of these cytokines to clinical parameters of periodontal disease. Levels were assessed: colony stimulating factor and granulocyte-macrophage colony-stimulating factor (GM-CSF), IL-1 $\beta$, IL-2, IL-4, IL-5, IL-6, IL-8, IL-10, interferon-c (INF-c) and TNF- $\alpha$, measured by enzyme linked immunosorbent assay multiplex. There were no statistically significant differences between groups for any cytokine measured. Found only weak positive association between salivary levels of IL-8 and pocket depth and bleeding on probing, and little negative correlation between the levels of salivary IL-10, attachment level and bleeding on probing. It is concluded, therefore, that the mean salivary cytokine levels could not directly diagnose periodontal disease.
In order to observe changes in markers of periodontal disease activity in response to treatment, Sexton et $\mathrm{al}^{27}$ made a longitudinal follow-up for 6 months, in a case-control study of patients with chronic periodontitis who received only oral hygiene instruction (OHI) and others who received SRP, combined with IHO. Saliva samples collected at 0,16 and 28 weeks were analyzed for IL-1 $\beta$, IL-8, MIP-1 $\alpha$, MMP-8, OPG and TNF. Clinical measures of periodontal disease were recorded in each patient return. OPG and TNF- $\alpha$ significantly decreased in both follow-up visits, regardless of treatment group. IL-1 $\beta$ and MMP-8 levels had decreased significantly since the beginning, but only in the group that received SRP. OPG, MMP-8 and MIP-1 $\alpha$ were significantly reduced in patients who responded to treatment, compared with those who did not respond. It is suggested that salivary levels of IL-1b, MMP-8, OPG and MIP- $1 \alpha$ reflect the severity of the disease, and subsequently, the response to periodontal treatment and may be useful for monitoring the status of longitudinal periodontal disease.

Aiming eliminate the varieties among the samples due to the differences between saliva samples from different individuals, one study also sought to compare these profiles of patients before and after periodontal treatment. Haigh et $\mathrm{al}^{25}$ carried out a quantitative proteomic analysis of saliva samples in order to identify changes in salivary proteome in the presence or absence of severe periodontitis, through two-dimensional electrophoresis. Nine male patients were enrolled, in which initial saliva sample was collected prior to treatment initiation and the second saliva sample was collected at the end of the treatment. The authors identified 15 proteins with altered response in the presence of periodontal disease, being the majority isotopes of S100:S100A8/A9/A6. Haptoglobin, prolactin secretory and proteins of the parotid proteins were also changed. When comparing the samples before and after the treatment, 15 proteins were identified with changes, and only S100A8, S100A9 and PSP were identified as altered in saliva only during periodontitis. Some proteins that have been associated with periodontal disease condition such as, some cytokines and MMPs cannot be quantified using this technique. This suggests that none of these proteins per se consists of a marker with sufficient power to determine disease activity. However, it is suggested by the authors that the identification of these markers may be used only to improve the disease diagnosis.

This inability to identify a single marker for periodontal disease, suggests the possibility of a clinical trial involving a combination of biomarkers, to identify novel molecules that can recognize the disease. These markers have been selected based on their role in the inflammatory process. An alternative currently being evaluated is proteomics and the changes in 
the proteomic profile in the presence of the disease. ${ }^{6}$ The clinical value of salivary proteomic biomarkers in the diagnosis of periodontal disease is still under experimental development, and is based on the molecular profile changes involved in inflammation, collagen degradation and bone loss resulting from periodontal disease activity. ${ }^{6,23}$

Assessment of salivary biomarkers for the diagnosis of gingivitis has already been proposed by analysis of proteomic profiles, comparing the profiles of patients with gingivitis to the profiles of healthy patients. It was found in one study the association of gingival inflammation with increased salivary levels of some immunoglobulins and keratins, and other peptides involved in the inflammatory process, as well as blood proteins, possibly due to spontaneous bleeding due to gingivitis. Some proteins are commonly detected in salivary proteomic analysis such as, mucin and histatin and may not, therefore, be associated with specific changes caused by gingivitis. Due to the ease and simplicity of clinical diagnosis of gingivitis this analyzes could be dispensable. However, their results can be useful for validating other methods that involve the specific diagnosis of periodontal disease activity. ${ }^{28}$

\section{DISCUSSION}

Many limitations are found for the use of salivary diagnostic. In order to be used the salivary biomarkers; there must be a relationship between levels of a given marker to be assessed for its presence in saliva and blood plasma. In addition, any amount of blood present in saliva due to gingival bleeding, for example, the salivary diagnosis could be compromised. ${ }^{5}$

Although in a previous study, ${ }^{23}$ the correlation with periodontal pathogens has been positive, it is stressed the need to assess to the longitudinally disease to obtain one or more markers that indicate the presence and activity of periodontal disease. Standardizing the saliva collection could also be useful for the comparative analysis of biomarkers. Especially considering the proposal of a rapid diagnostic test, the standardized schedule of collections, for example, could avoid possible biases due to metabolic changes of the individuals.

Although there are studies that compare the profile of the same patient before and after treatment, there is no comparison with the condition of absence of any treatment, as it is considered the OHI a fundamental part of basic periodontal treatment. The fact that the patient consciously participate in a survey, contribute to the improvement of clinical parameters of the disease. Possibly, the major differences between the groups and also a more defined relationship with some biomarkers would be found if the patients had been assessed longitudinally in comparison with untreated patients. ${ }^{27}$
Furthermore, Teles et $\mathrm{al}^{26}$ stated that the amount of cytokines present in the tested samples could not represent the true, due to the influence of several factors, or that the representative amount may be very low and cannot be detected by the means used, suggesting that new approaches could be used in an attempt to establish this association. However, the quantities of inflammatory cytokines that can be detected in the saliva, although different between healthy individuals from periodontitis patients, are always very low, suggests that such detection may not be sufficient for determining periodontal disease. There are still differences between the sites that are diagnosed with periodontal disease as the activity and severity of disease.

Other difficulties in dealing with biological tests aimed possible clinical applications include the need to maintain the integrity and stability of the molecules to be evaluated from the saliva collected. DNA and RNA as well as proteins, are unstable molecules with short half-life. Protocols have been studied in order to develop techniques to preserve the stability of DNA, RNA and proteins from saliva, without significant changes in the identification and quantification of these molecules in the samples. ${ }^{29,30}$ Such protocols, however, still require different preparation for use, besides the high cost of specific reagents.

The proteomic analysis has been presented as advantageous over other techniques for protein identification by the possibility to identify different profiles of many different protein targets. However, the identification of isoforms of proteins and specific cytokines can also be made by enzyme immunoassays such as ELISA or Western blot, since use is made of primary antibody that recognizes specific epitopes of these isoforms. A disadvantage regarding the use of proteomics is that proteins present in saliva may be partially degraded to peptide fragments, which cannot be identified and/or related to any particular protein or cytokine. In addition, high complexity and high cost make impossible to use these methods today.

As for biomarkers, was not found until now a single marker that could clearly identify the presence and activity of periodontitis. ${ }^{7,31}$ Studies have been expected to be prone to develop a rapid and effective test, based not on a single biomarker, but a number of these, in order to accurately identify changes caused by the complex course of periodontal disease. In addition, saliva is subject to numerous changes in its composition due to local factors and systemic changes, and could not yet be considered a sufficient diagnostic tool to determine the activity of periodontal diseases.

\section{CONCLUSION}

The scientific literature has limitations in its methodology, and these interfere and hinder standardization among groups, 
casting doubt on the credibility of the results. Therefore, until the present time, saliva could not be considered as a means of diagnosis sufficient for periodontal disease activity, as is subject to numerous changes in its composition due to local factors such as systemic disorders. However, periodontal treatment based on assessing the levels of salivary biomarkers, emerging as a promising method in near future and will become an integral part of the evaluation of periodontal health.

\section{REFERENCES}

1. de Molon RS, de Avila ED, Cirelli JA. Host responses induced by different animal models of periodontal disease: a literature review. J Investig Clin Dent 2013;4:211-218.

2. de Molon RS, de Avila ED, Nogueira AV, de Souza JA, AvilaCampos MJ, de Andrade CR, Cirelli JA. Evaluation of the host response in various models of induced periodontal disease in mice. J Periodontol 2013 Jun 27. [Epub ahead of print].

3. Armitage GC, Research, Science and Therapy Committee of the American Academy of Periodontology. Diagnosis of periodontal diseases. J Periodontol 2003;74(8):1237-1247.

4. Lang NP, Tonetti MS. Periodontal diagnosis in treated periodontitis. Why, when and how to use clinical parameters. J Clin Periodontol 1996;23(3 Pt 2):240-250.

5. Malamud D. Saliva as a diagnostic fluid. Dent Clin North Am 2011;55(1):159-178.

6. Zhang L, Henson BS, Camargo PM, Wong DT. The clinical value of salivary biomarkers for periodontal disease. Periodontol 2000 2009;51:25-37.

7. Kaufman E, Lamster IB. Analysis of saliva for periodontal diagnosis--a review. J Clin Periodontol 2000;27(7):453-465.

8. Olayanju OA, Rahamon SK, Joseph IO, Arinola OG. Salivary immunoglobulin classes in Nigerians with periodontitis. J Contemp Dent Pract 2012;13(2):163-166.

9. Agha-Hosseini F, Mirzaii-Dizgah I, Rahimi A, Seilanian-Toosi M. Correlation of serum and salivary CA-125 levels in patients with breast cancer. J Contemp Dent Pract 2009;10(6):E001-008.

10. Balan JJ, Rao RS, Premalatha BR, Patil S. Analysis of tumor marker CA-125 in saliva of normal and oral squamous cell carcinoma patients: a comparative study. J Contemp Dent Pract 2012;13(5):671-675.

11. Jagtap K, Baad RK. Estimation of salivary nitric oxide in recurrent aphthous ulcer and oral lichen planus patients with its clinical significance. J Contemp Dent Pract 2012;13(5):623-626.

12. Qvarnstrom M, Janket S, Jones JA, Nuutinen P, Baird AE, Nunn ME, Van Dyke TE, Meurman JH. Salivary lysozyme and prevalent hypertension. J Dent Res 2008;87(5):480-484.

13. Floriano PN, Christodoulides N, Miller CS, Ebersole JL, Spertus J, Rose BG, Kinane DF, Novak MJ, Steinhubl S, Acosta S, et al. Use of saliva-based nano-biochip tests for acute myocardial infarction at the point of care: a feasibility study. Clin Chem 2009;55(8):1530-1538.

14. Rao PV, Reddy AP, Lu X, Dasari S, Krishnaprasad A, Biggs E, Roberts CT, Nagalla SR. Proteomic identification of salivary biomarkers of type-2 diabetes. J Proteome Res 2009;8(1): 239-245.

15. Cummins MJ, Papas A, Kammer GM, Fox PC. Treatment of primary Sjogren's syndrome with low-dose human interferon alfa administered by the oromucosal route: combined phase III results. Arthritis Rheum 2003;49(4):585-593.
16. Buduneli E, Vardar-Sengul S, Buduneli N, Atilla G, Wahlgren J, Sorsa T. Matrix metalloproteinases, tissue inhibitor of matrix metalloproteinase-1, and laminin-5 gamma-2 chain immunolocalization in gingival tissue of endotoxin-induced periodontitis in rats: effects of low-dose doxycycline and alendronate. J Periodontol 2007;78(1):127-134.

17. Ingman $T$, Tervahartiala $T$, Ding $Y$, Tschesche H, Haerian A, Kinane DF, Konttinen YT, Sorsa T. Matrix metalloproteinases and their inhibitors in gingival crevicular fluid and saliva of periodontitis patients. J Clin Periodontol 1996;23(12):11271132.

18. Sorsa T, Tjaderhane L, Salo T. Matrix metalloproteinases (MMPs) in oral diseases. Oral Dis 2004;10(6):311-318.

19. Miller CS, King CP Jr, Langub MC, Kryscio RJ, Thomas MV. Salivary biomarkers of existing periodontal disease: a crosssectional study. J Am Dent Assoc 2006;137(3):322-329.

20. Herr AE, Hatch AV, Throckmorton DJ, Tran HM, Brennan JS, Giannobile WV, Singh AK. Microfluidic immunoassays as rapid saliva-based clinical diagnostics. Proc Natl Acad Sci U S A 2007;104(13):5268-5273.

21. Yoshie H, Tai H, Kobayashi T, Oda-Gou E, Nomura Y, Numabe Y, Ito K, Kurihara H, Kamoi K. Salivary enzyme levels after scaling and interleukin-1 genotypes in Japanese patients with chronic periodontitis. J Periodontol 2007;78(3):498-503.

22. Kinane DF, Darby IB, Said S, Luoto H, Sorsa T, Tikanoja $\mathrm{S}$, Mantyla P. Changes in gingival crevicular fluid matrix metalloproteinase- 8 levels during periodontal treatment and maintenance. J Periodontal Res 2003;38(4):400-404.

23. Ramseier CA, Kinney JS, Herr AE, Braun T, Sugai JV, Shelburne CA, Rayburn LA, Tran HM, Singh AK, Giannobile WV. Identification of pathogen and host-response markers correlated with periodontal disease. J Periodontol 2009;80(3):436-446.

24. Wu Y, Shu R, Luo LJ, Ge LH, Xie YF. Initial comparison of proteomic profiles of whole unstimulated saliva obtained from generalized aggressive periodontitis patients and healthy control subjects. J Periodontal Res 2009;44(5):636-644.

25. Haigh BJ, Stewart KW, Whelan JR, Barnett MP, Smolenski GA, Wheeler TT. Alterations in the salivary proteome associated with periodontitis. J Clin Periodontol 2010;37(3):241-247.

26. Teles RP, Likhari V, Socransky SS, Haffajee AD. Salivary cytokine levels in subjects with chronic periodontitis and in periodontally healthy individuals: a cross-sectional study. J Periodontal Res 2009;44(3):411-417.

27. Sexton WM, Lin Y, Kryscio RJ, Dawson DR 3rd, Ebersole JL, Miller CS. Salivary biomarkers of periodontal disease in response to treatment. J Clin Periodontol 2011;38(5):434-441.

28. Goncalves Lda R, Soares MR, Nogueira FC, Garcia C, Camisasca DR, Domont G, Feitosa AC, Pereira Dde A, Zingali RB, Alves G. Comparative proteomic analysis of whole saliva from chronic periodontitis patients. J Proteomics 2010;73(7):1334-1341.

29. Park NJ, Yu T, Nabili V, Brinkman BM, Henry S, Wang J, Wong DT. RNA protect saliva: An optimal room temperature stabilization reagent for the salivary transcriptome. Clin Chem 2006;52(12):2303-2304.

30. Jiang J, Park NJ, Hu S, Wong DT. A universal preanalytic solution for concurrent stabilization of salivary proteins, RNA and DNA at ambient temperature. Arch Oral Biol 2009;54(3):268-273.

31. Ozmeric N. Advances in periodontal disease markers. Clin Chim Acta 2004;343(1-2):1-16. 
Use of Salivary Biomarkers for Diagnosis of Periodontal Disease Activity: A Literature Review

\section{ABOUT THE AUTHORS}

\section{Fernanda Regina Godoy Rocha}

PhD Student, Department of Diagnosis and Surgery, School of Dentistry, Univ Estadual Paulista - UNESP, Araraquara, São Paulo, Brazil

\section{Rafael Scaf de Molon}

PhD Student, Department of Diagnosis and Surgery, School of Dentistry, Univ Estadual Paulista - UNESP, Araraquara, São Paulo, Brazil

Correspondence Address: RuaHumaitá, 1680, 14801-903, Araraquara, São Paulo, Brazil, Phone: +55 (16)3114-1247, e-mail:molon.foar@yahoo.com.br

\section{Mateus Rodrigues Tonetto}

PhD Student, Department of Restorative Dentistry, School of Dentistry, Univ Estadual Paulista - UNESP, Araraquara, São Paulo, Brazil

\section{Shelon Cristina Souza Pinto}

Assistant Professor, Department of Dentistry, University of Ponta Grossa State, Ponta Grossa, Paraná, Brazil

\section{Eduardo Mariscal Munoz}

PhD Student, Department of Dental Materials and Prosthodontics School of Dentistry, Univ Estadual Paulista - UNESP, Araraquara São Paulo, Brazil

\section{Jose Eduardo Cesar Sampaio}

Adjunct Professor, Department of Diagnosis and Surgery, School of Dentistry, Univ Estadual Paulista - UNESP, Araraquara, São Paulo Brazil 